\title{
Síndrome de Down y envejecimiento: una nueva situación que afrontar
}

\section{Down syndrome and ageing: facing a new situation}

\section{Palabras clave}

Síndrome de Down, alzhéimer, demencia, evaluación, buenas prácticas, asociación

\section{Keywords}

Down's syndrome, Alzheimer's disease, dementia, assessment, good practices, association

\section{Introducción}

Envejecer es un proceso natural para todas las personas, pero no deja de ser una conquista para aquellas que tienen síndrome de Down. En la actualidad la esperanza de vida de las personas con este síndrome se sitúa en torno a los 60 años (Bittles y Glasson, 2004). Tal logro viene derivado del estudio y del conocimiento exhaustivo de la realidad y necesidades del colectivo, dando lugar a una mejora exponencial de su calidad de vida y, muy especialmente, a una mejor y más especializada atención en el campo de la salud, sobre todo en las primeras etapas de la vida. Por supuesto, también por las mejoras en la atención social a través de entidades de apoyo, mediante las que se consiguió prevenir la dependencia y aumentar las cuotas de autonomía de las personas pertenecientes a este colectivo.

Vivir mejor y contar con una mejor salud ha hecho posible que, a día de hoy, las personas con síndrome de Down hayan conquistado una nueva etapa vital: la vejez.

\section{María Muíño Filgueira}

$<$ vidaadulta@teimadownferrol. org >

Teima Down Ferrol. España

\section{Sandra Peña Díaz}

$<$ vida.adulta@downxuntos.org>

Down Pontevedra Xuntos. España

\section{Alba Iglesias López}

<comunicación@downgalicia. org>

Federación Down Galicia. España

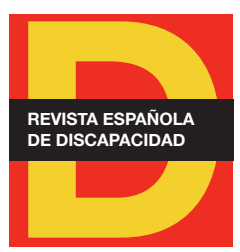

Para citar:

Muíño, M. et al. (2019): "Síndrome de Down y envejecimiento: una nueva situación que afrontar". Revista Española de Discapacidad, 7 (II): 157-164.

Doi: <https://doi.org/10.5569/23405104.07.02.09> 


\section{Síndrome de Down, envejecimiento y enfermedad de Alzheimer}

Las personas con síndrome de Down experimentan un proceso de envejecimiento acelerado, por lo que a partir de los 40-45 años comienzan a presentar rasgos típicos de la población envejecida (Nakamura y Tanaka, 1998; Presson et al., 2013).

De este manera, es necesario llevar a cabo un exhaustivo control médico que tenga en cuenta los problemas clínicos desde que son personas adultas hasta que envejecen, ya que, por un lado, será necesario adelantar la edad de aplicación de ciertos protocolos para pruebas diagnósticas y, por otro, será de especial relevancia a la hora de elaborar un correcto diagnóstico de demencia.

Como ejemplo, en la actualidad nos encontramos con dos perfiles de personas usuarias en las entidades de la Federación Down Galicia:

- $\quad$ Personas que son usuarias de los servicios de las entidades Down desde pequeñas y que están envejeciendo en los servicios con un perfil activo.

- $\quad$ Personas que entran a formar de las entidades Down en etapa adulta al fallecer sus progenitores, casi siempre sin escolarizar y con un perfil más dependiente.

Desde las entidades Down de Galicia creemos firmemente en que el modelo de vida inclusivo que promovemos, que garantiza la participación en entornos y servicios comunitarios, tendrá una repercusión positiva en su envejecimiento.

Por otra parte, también está constatado que existe una estrecha relación entre el síndrome de Down y la enfermedad de Alzheimer (Glasson et al., 2014; Hermans y Evenhuis, 2014). A través de la realización de estudios se pudo comprobar que casi todas las personas con síndrome de Down de más de 40 años tenían desarrolladas características neuropatológicas propias de esta enfermedad (Doran et al., 2017).

Esta circunstancia está relacionada con la alteración genética que origina el síndrome de Down; es decir, con la presencia de material genético extra en el cromosoma 21. El par 21 tiene relación con los procesos de envejecimiento y con la producción de la proteína beta-amiloide que, al acumularse en el cerebro, da lugar a las placas seniles asociadas a la enfermedad de Alzheimer. Estas placas comienzan a aparecer en torno a los 8 años de edad, si bien los síntomas no se manifiestan hasta pasados 30 años de la formación de las mismas (Flórez, 2010).

Es por ello que parece que tener más carga genética en el par 21 influye para que las personas con síndrome de Down envejezcan antes de tiempo y tengan más probabilidad de desarrollar esta enfermedad.

Los estudios más recientes afirman que entre un $50 \%$ y un $70 \%$ de las personas con síndrome de Down mayores de 60 años padecerán la enfermedad de Alzheimer (Strydom et al., 2010). El promedio de edad de la aparición de la enfermedad es, por consiguiente, 20 años más baja que en la población general (Nakamura y Tanaka, 1998; Presson et al., 2013). Además, el avance de la enfermedad es más rápido y se sitúa como la mayor causa de mortalidad en las personas adultas con síndrome de Down (Fortea, 2019). 
Por lo tanto, esta longevidad, siendo un gran logro, es también un gran reto. Se trata de una nueva realidad que pone en evidencia la existencia de una serie de carencias importantes para afrontar esta situación. Es fundamental tomar conciencia de que las personas con síndrome de Down pueden presentar en su envejecimiento diferentes patologías que precisan ser detectadas, diagnosticadas y tratadas igual que al resto de la población. Para tal fin es necesario anticiparse y tener datos suficientes de los factores biológicos, psicológicos y sociales para, de este modo, contribuir a diagnósticos más fiables de las patologías ligadas al envejecimiento.

A día de hoy, el proceso de envejecimiento de las personas con síndrome de Down está lleno de interrogantes, por lo que es necesario convertirlo en un objeto de estudio que permita conocer lo máximo posible esta realidad, para ser capaz de predecir y prepararse para afrontar de la mejor manera posible las necesidades y demandas de las personas mayores con síndrome de Down y de sus familias. Así se garantizará su calidad de vida en esta etapa y se preservará su derecho a participar y beneficiarse de los recursos especializados en la atención a mayores ya existentes en la comunidad.

\section{La experiencia de una entidad afrontando el envejecimiento}

La Federación Down Galicia desarrolla su labor acompañando y dando soporte a sus entidades miembro en la actividad de apoyo a las personas con síndrome de Down y otras discapacidades intelectuales para el libre ejercicio de sus derechos y para el desarrollo de sus proyectos de vida en el marco inclusivo de los contextos sociales a los que pertenecen.

Durante sus 20 años de historia, fueron muchos los proyectos desarrollados y los logros y avances conseguidos a favor de una mayor calidad de vida en todas las etapas vitales. Con todo, el paso del tiempo siempre va trayendo nuevas perspectivas y realidades. Así, en las distintas entidades comenzó a aumentar el número de personas con síndrome de Down envejecidas, lo que trajo consigo muchas incógnitas para las que no había respuesta. La federación se encontró de pronto ante un nuevo reto, prestar los apoyos adecuados para afrontar esta nueva etapa vital.

De este modo, y teniendo siempre presente la Convención sobre los Derechos de las Personas con Discapacidad de la Organización de las Naciones Unidas aprobada en el año 2006, en enero de 2013 se decide crear la Comisión de envejecimiento de Down Galicia.

Esta comisión, constituida inicialmente como grupo de trabajo a nivel interno, está integrada por profesionales de la Federación Down Galicia y de todas las entidades que conforman la misma y su finalidad está centrada en determinar cómo abordar el envejecimiento de sus personas usuarias y darle una respuesta idónea a sus nuevas necesidades.

La Comisión de envejecimiento de Down Galicia parte de una situación de escaso conocimiento ante una situación novedosa y, en un primer momento, se decide realizar un análisis de la realidad existente mediante la que se detectan una serie de dificultades que será necesario abordar: 
- Presencia de cambios a nivel conductual, funcional y/o cognitivo en ciertas personas usuarias de edad más avanzada, pero aún relativamente jóvenes.

- Elevado nivel de envejecimiento a edades relativamente tempranas, con alta incidencia de complicaciones a nivel de salud.

- Dificultades para la realización de diagnósticos por un elevado impacto del "efecto eclipsador" de la discapacidad intelectual. Esto es, cambios y patología asociadas directamente al síndrome de Down, obviando otros aspectos importantes para un idóneo diagnóstico.

- Ausencia de diagnósticos en casos en los que se evidenció la presencia de la enfermedad de Alzheimer, excluyendo de este modo a estas personas de los recursos asistenciales disponibles en la comunidad para su atención.

Una vez analizadas estas dificultades, la Comisión de envejecimiento de la federación consensuó una serie de necesidades para poder responder de forma idónea a esta situación:

- Identificar procedimientos para detectar situaciones de alerta de manera precoz que permitan disponer de datos objetivos y fiables.

- Conocer estrategias para abordar la etapa del envejecimiento desde un enfoque normalizador e inclusivo.

- Difundir y sensibilizar a la comunidad médica sobre la realidad del envejecimiento y el síndrome de Down.

Desde un primer momento, para la elaboración de un modelo adecuado de atención a las personas mayores con síndrome de Down, la comisión apostó por la búsqueda continua de alianzas, firmándose sendos convenios de colaboración con la Sociedad Galega de Xeriatría e Xerontoloxía (SGXX) y con la Federación Alzheimer Galicia (FAGAL).

Asimismo, dentro de la apuesta por la formación continua del equipo de profesionales, destaca la asistencia de los miembros de la comisión al XXVI Congreso Internacional de Geriatría y Gerontología, celebrado en Pontevedra en junio de 2014, y a las XVI Jornadas Internacionales de la Fundació Catalana de Síndrome de Down (FCSD) en noviembre de 2015, donde referentes mundiales en la investigación del síndrome de Down y la enfermedad de Alzheimer constataron la relación entre ambas y evidenciaron la necesidad de contar con instrumentos adaptados para su diagnóstico.

Así, en el año 2016, la Comisión inicia lo que llama Estudio del envejecimiento de las personas con síndrome de Down en el que se enmarca, por una parte, la elaboración del Protocolo de evaluación y seguimiento del envejecimiento y cuyo éxito se fundamenta en la coordinación de las entidades de Down Galicia con los profesionales del ámbito socio-sanitario. Este protocolo, que tiene por objetivo compensar las dificultades existentes para identificar aquellos signos de alerta que indiquen cambios en el estado global de la salud de las personas con síndrome de Down y contribuir a su valoración, establece una serie de instrumentos para la recogida y posterior análisis de datos longitudinales referidos a estas personas en su etapa adulta, que contribuyen a un mejor conocimiento de los procesos de envejecimiento y de apoyo ante la necesidad de un diagnóstico. 
Por otra parte, se crean distintas encuestas enmarcadas en el mismo estudio con el fin de identificar las necesidades de las personas usuarias y de sus familias en los procesos de envejecimiento. En base a toda esta información, el objetivo último del estudio será definir las líneas de actuación y los recursos de apoyo necesarios para dar respuesta a las necesidades detectadas.

En ese mismo año, la Comisión se incorpora al Grupo de Demencias de la Federación Alzheimer Galicia (FAGAL) y varios de sus miembros participaron como ponentes en las XIV Jornadas Interdisciplinares que esta federación organizó en el mes de octubre.

En marzo de 2017, la Comisión de envejecimiento participa en el Foro Interdisciplinar en Demencias de A Coruña (FIEDAC) y en noviembre del mismo año se une a la Red Galega de Investigación en Demencias como entidad invitada, pudiendo asistir al primer foro organizado por esta red y participando como entidad ponente en el segundo, celebrado en enero de 2018.

Desde el pasado año, la Comisión está colaborando con las universidades de Santiago de Compostela (USC) y Vigo (UVIGO) en la definición de formatos de descarga y análisis de datos, así como presentando en varios másteres una unidad didáctica que tiene por objetivo dar a conocer las características del proceso de envejecimiento de las personas con síndrome de Down y proporcionar estrategias básicas para la atención y la relación con este colectivo.

Asimismo, se mantienen reuniones de manera continuada con especialistas médicos de las distintas áreas sanitarias de Galicia en la busca de aliados que añadan valor al Protocolo de evaluación y seguimiento del envejecimiento de las personas con síndrome de Down.

A finales del pasado año, se elaboró una Guía de salud para personas adultas con síndrome de Down (Down Galicia, 2018). Este documento es un instrumento de apoyo a las familias y a los profesionales sanitarios que pretende dar respuesta a las necesidades de control de la salud y seguimiento preventivo que necesitan las personas con síndrome de Down mayores de 18 años.

\section{Objetivos de trabajo de la Comisión de envejecimiento}

La Convención sobre los Derechos de las Personas con Discapacidad reafirma que todas las personas con independencia de sus características personales deben poder disfrutar de todos los derechos humanos y libertades fundamentales. Entiende la discapacidad como un concepto que evoluciona, y esa evolución dependerá de la interacción de las características de la persona con discapacidad con los obstáculos que presenta el entorno (barreras físicas y actitudes imperantes) y que impiden su participación en la sociedad. Cuantos más obstáculos haya, mayores serán las limitaciones para vivir con plenitud.

Son numerosas las barreras existentes para la participación y el ejercicio de los derechos de las personas con discapacidad. La mayor parte de estas barreras poco o nada tienen que ver con las dificultades que pueda implicar la condición de discapacidad. 
La Comisión de envejecimiento de Down Galicia considera que el envejecimiento debe ser abordado desde la metodología de la Atención Integral Centrada en la Persona (AICP) (Rogers, 1942; Kitwood, 1997) y el enfoque del envejecimiento activo. El trabajo llevado a cabo, que tiene como finalidad el fomento de la inclusión en esta etapa promoviendo la participación en los recursos comunes, irá enfocado al cumplimiento de los siguientes objetivos:

- Avanzar en el conocimiento de las necesidades de las personas usuarias, escuchando sus voces y teniendo en cuenta sus propuestas.

- Promover la visibilidad y realidad del colectivo.

- Ofrecer y demandar alternativas inclusivas.

- Crear vínculos con los y las profesionales y con los recursos comunitarios.

- Reclamar que se contemplen las particularidades del colectivo en el acceso a recursos dirigidos la mayores.

- Reivindicar avances en los procesos de diagnóstico de trastornos ligados al envejecimiento.

Por lo tanto, con esta nueva realidad es necesario poner en marcha programas de atención integral en el envejecimiento, en los que se tengan en cuenta las características particulares del mismo en las personas con síndrome de Down y otras discapacidades intelectuales.

Las entidades miembro de la federación se encuentran inmersas en estos proyectos, que se centran en el diseño de actividades que promuevan las condiciones necesarias para la consecución de mejoras en todos los ámbitos de la calidad de vida y bienestar de la persona, partiendo del respeto pleno a su dignidad y derechos, de sus intereses y preferencias, al tiempo que se cuenta con su participación efectiva.

\section{Un largo camino por recorrer con un claro objetivo}

Tras el trabajo llevado a cabo hasta el momento actual, la Comisión de envejecimiento de la Federación Down Galicia presenta una serie de conclusiones sobre la relación entre el envejecimiento y el síndrome de Down.

Por un lado, un envejecimiento prematuro y acelerado implica que las medidas de prevención y detección de signos de envejecimiento comiencen a aplicarse a edades tempranas. Entre estas medidas, el cuidado de la salud y la prevención de la dependencia y la salud mental deben ocupar un espacio prioritario.

Por otro lado, el desconocimiento de la realidad de las personas con síndrome de Down en esta etapa vital genera muchas dificultades para la realización de diagnósticos que diferencien adecuadamente entre comportamientos o conductas propias de la persona con discapacidad intelectual, de otros que puedan indicar la presencia de alguna enfermedad mental o deterioro cognitivo. Hace falta, por lo tanto, introducir entre las 
medidas a llevar a cabo, una recogida de información sistematizada, objetiva y periódica que contribuya a la elaboración de un perfil de funcionamiento que ayude a detectar cambios significativos que puedan ser indicadores de la presencia de una enfermedad o trastorno.

Asumir que las personas con síndrome de Down vivirán muchos años implica introducir las medidas necesarias que contribuyan a alcanzar esta etapa vital con la mayor calidad de vida posible y que permitan planificar este momento de la vida de la forma más idónea. 


\section{Referencias bibliográficas}

Bittles, A.H. y Glasson, E.J. (2004): "Clinical, social and ethical implications of changing life expectancy in Down syndrome”. Developmental Medicine and Child Neurology, 46: 282-286.

Down Galicia (2018): Guía de Saúde para persoas adultas coa síndrome de Down (en línea). <https://downgalicia. org/wp-content/uploads/2019/06/Triptico-Saude-.pdf>, acceso 3 de septiembre de 2019.

Doran, E. et al. (2017). "Down Syndrome, partial trisomy 21, and absence of Alzheimer's disease: the role of APP". Journal of Alzheimer's Disease, 56: 459-470.

Flórez, J. (2010): “Enfermedad de Alzheimer y síndrome de Down”. Revista síndrome de Down, 27: 63-76.

Fortea, J. (2019): Enfermedad de Alzheimer en el síndrome de Down. LCR, RM, EEG y PET - Estudios multimodales (en línea). <http://zink.es/Simposium20_Site/memoria/cast/DrFortea>, acceso 3 de septiembre de 2019.

Glasson, E.J. et al. (2014): "The triple challenges associated with age-relates comorbidities in Down syndrome". Journal of Intellectual Disability Research, 58: 393-398.

Hermans, H. y Evenhuis, H.M. (2014): "Multimorbidity in older adults with intellectual disabilities". Research in Developmental Disabilities, 35: 776-783.

Kitwood, T. (1997): Dementia reconsidered: The person comes first. Buckingham: Open University Press.

Nakamura, E. y Tanaka, S. (1998): "Biological ages of adult men and women with Down's syndrome and its changes with aging". Mechanisms of Ageing and Development, 105: 89-103.

Naciones Unidas (2006): Convención Internacional sobre los Derechos de las Personas con Discapacidad. Nueva York: ONU.

Presson, A.P. et al. (2013): "Current estimate of Down syndrome population prevalence in the United States". The journal of Pediatrics, 163 (4): 1163-1168.

Rogers, C. (1942): Counselling and psychotherapy. Boston: Houghton Mifflin.

Strydom, A. et al. (2010): "Dementia in older adults with intellectual disabilities - Epidemiology, presentation, and diagnosis". Journal of Policy and Practice in Intellectual Disabilities, 7: 96-110. 